\title{
Assessment of choriocapillary blood flow changes in response to half-dose photodynamic therapy in chronic central serous chorioretinopathy using optical coherence tomography angiography
}

\author{
Juejun Liu, Changzheng Chen*, Lu Li, Yishuang Xu, Zuohuizi Yi, Lu He and Hongmei Zheng
}

\begin{abstract}
Background: Optical coherence tomography angiography (OCTA) is a newly developed imaging quantitative technique for analysis of choriocapillaris (CC) flow changes, thereby exploring the pathological mechanism of chronic central serous chorioretinopathy (CCSC) and the therapeutic effects of photodynamic therapy (PDT). In this study, we sought to quantify the blood flow changes in CC of CCSC patients receiving half-dose PDT using OCTA.

Methods: A total of 28 affected eyes and 24 unaffected eyes of 26 CCSC patients receiving half-dose PDT, and 40 eyes of 20 healthy gender- and age-matched subjects were retrospectively enrolled in this study. The proportion of total areas of flow signal voids (FSV, \%) in CC level of OCTA was assessed in both eyes of the CCSC patients at baseline and repeated in multiple sections at 1-week, 1-month, 3-month and 6-month intervals after PDT. In addition, the CC patterns in response to PDT at early stage and the subsequent morphologic changes were qualitatively documented using OCTA.

Results: For affected eyes, FSV at 6-m follow-up was significantly lower than that at 1-m follow-up $(p=0.036)$. When compared to normal control eyes, FSV in affected eyes was significantly higher at $1-m, 3-m$ and $6-m$ followup $(p<0.05$ for all), and FSV in unaffected eyes was significantly higher at baseline, 1-w, 1-m and 3-m follow-up $(p<0.05$ for all). Three CC patterns of early response to PDT were identified, including signs of recovery with more even flow signals, transient appearance of worse ischemia and secondary neovascularization within CC level.
\end{abstract}

Conclusion: Abnormal CC flow attenuation remains in completely resolved eyes of CCSC patients treated with halfdose PDT.

Keywords: Chronic, Central serous Chorioretinopathy, Optical coherence tomography angiography, Photodynamic therapy

*Correspondence: whuchenchzh@163.com

Eye Center, Renmin Hospital of Wuhan University, Wuhan, China

(c) The Author(s). 2020 Open Access This article is licensed under a Creative Commons Attribution 4.0 International License, which permits use, sharing, adaptation, distribution and reproduction in any medium or format, as long as you give appropriate credit to the original author(s) and the source, provide a link to the Creative Commons licence, and indicate if changes were made. The images or other third party material in this article are included in the article's Creative Commons licence, unless indicated otherwise in a credit line to the material. If material is not included in the article's Creative Commons licence and your intended use is not permitted by statutory regulation or exceeds the permitted use, you will need to obtain permission directly from the copyright holder. To view a copy of this licence, visit http://creativecommons.org/licenses/by/4.0/ The Creative Commons Public Domain Dedication waiver (http://creativecommons.org/publicdomain/zero/1.0/) applies to the data made available in this article, unless otherwise stated in a credit line to the data. 


\section{Background}

With the development of fundus imaging technology, the potential mechanism of choroidal hemodynamic changes in central serous chorioretinopathy (CSC) has been elucidated using indocyanine green angiography (ICGA) and enhanced depth imaging optical coherence tomography (EDI-OCT) [1]. Choriocapillaris (CC) ischemia processes, along with increasing hydrostatic pressure from choroidal pachyvessels, could result in focal or defused retinal pigment epithelium (RPE) barrier breakdown and subsequent serous retinal detachment (SRD) [1-3]. It has been reported that photodynamic therapy (PDT), with modified parameters, has a long-term efficacy and safety profile in chronic CSC (CCSC) therapy, with fewer complications and greater improvements in vision, subretinal fluid (SRF) and subfoveal choroidal thickness (SFCT) [1, 4]. As demonstrated by previous pathological and angiographic studies, PDT can cause short-term CC hypoperfusion and long-term choroidal vascular remodeling in the treatment of CSC [5]. Assessment of $\mathrm{CC}$ in vivo could greatly help understand the pathological mechanism of CSC and the therapeutic effects of PDT [6, 7].

Optical coherence tomography angiography (OCTA) is a newly developed imaging technique, which provides new insights into CC perfusion and vasculature patterns over the selected layers free from dye injection, and greatly compensates for the deficiencies of OCT and ICGA in the observation of CC $[7,8]$. Several OCTA-related studies reported irregular flow patterns of $\mathrm{CC}$ in patients with CSC, including CC dilatation with increasing flow signals $[9,10]$, and focal or defused dark areas with flow signal voids (FSV) [7, 9], which were associated with the changes in ICGA. Various binary quantifications of OCTA imaging with objective data on various parameters further confirmed the attenuation of CC in CSC [8, $11,12]$. Previously, we have qualitatively documented that $97 \%(32 / 33)$ of eyes diagnosed with CCSC returned to exhibiting relatively normal distribution of fine particles in CC layer of OCTA at 3-m follow-up after half-dose PDT [13]. Nassisi M, et, al. reported that the CC vessel density at 1-m follow-up was even higher than the baseline value analyzed using an alternative binary approach [14], which however did not exclude the shadowing artifacts of SRF at enrollment. This present study was designed to assess CC blood flow changes in the affected eyes of CCSC patients in response to half-dose PDT assessed at early and long-term follow-ups using OCTA. The results were compared with those of the contralateral unaffected eyes as well as the healthy control eyes to explore the possible differences between them in response to half-dose PDT.

\section{Methods}

Patients with CCSC and healthy gender- and agematched subjects, from November 2017 to November 2018, were retrospectively enrolled in this study, and all participants provided written informed consent. CCSC is defined [10] as exhibition of visual acuity symptoms for at least 6 months along with documented clinical features of CSC, including macular SRF, RPE changes of leakage and hyperfluorescent alterations detected by OCT and FFA/ICGA. All patients in this study were subjected to half-dose PDT with verteporfin infusion (3 $\mathrm{mg} / \mathrm{m}^{2}$ of body surface area), and the laser light (689 $\mathrm{nm} ; 50 \mathrm{~J} / \mathrm{cm}^{2}$ for $83 \mathrm{~s}$ ) was projected at the area of hyperpermeability in ICGA for $15 \mathrm{~min}$ after the start of verteporfin infusion. PDT was performed by an experienced ophthalmologist. The patients with eyes subjected to any previous treatment or exhibiting myopia more than 3.0 diopters, as well as those suffering from other diseases like PCV and neovascular maculopathy (eg, agerelated macular degeneration, pathologic myopia, and idiopathic $\mathrm{CNV}$ ) were excluded from the study upon clinical examination.

All patients had received thorough ophthalmic examinations prior to PDT treatment, including bestcorrected visual acuity (BCVA) assessment, fundus fluorescein angiography (FFA), ICGA (HRA2; Heidelberg Engineering, Heidelberg, Germany), fundus photography (Zeiss, Oberkochen, Germany), spectral-domain OCT (SD-OCT) and OCTA (RTVue XR AngioVue Version 2017.1; Optovue Inc., Fremont, CA, USA). Quantitative assessments were performed at baseline and repeated in multiple sections at 1-week, 1-month, and 3-month and 6-month intervals after PDT treatment. These assessments included central macular thickness (CMT) in the mode of retina map of SD-OCT, subfoveal choroidal thickness (SFCT) in the mode of Enhanced HD Line (Enhanced HD-OCT) of SD-OCT, and the proportion of total areas of FSV (\%) in OCTA using binary processing. Healthy gender- and age-matched subjects without ocular diseases were enrolled at a single visit and examinations of indirect ophthalmoscopy, BCVA, SD-OCT and OCTA were performed. Of those healthy subjects, those with systemic diseases like diabetes, hypertension, myopia more than 3.0 diopters and ocular media opacity that could affect the scan quality of OCTA were excluded from the study.

Enhanced HD-OCT was performed automatically focusing on the macular fovea with a horizontal single-line scanning, the scanning was repeated more than twice and the best one (the clearest image with a signal strength index of more than $70 \%$ and without eye movement artifacts) was selected. SFCT is defined as the vertical distance between the outside boundary of RPE and the choroidal-scleral interface determined by averaging 
the values obtained at three positions: the subfovea area, $750 \mu \mathrm{m}$ nasal and $750 \mu \mathrm{m}$ temporal to the fovea. OCTA, using a light source of $840 \mathrm{~nm}$ and an A-scan rate of 70, 000 scans per second, was incorporated with splitspectrum amplitude-decorrelation angiography (SSADA) algorithm to extract angiographic information by quantifying the decorrelation of the OCT reflectance between the two consecutive B-scans, each containing 304 Ascans at each location on the retina [15]. $3 \times 3 \mathrm{~mm}^{2}$ OCTA scanning was acquired centering on the fovea with eye tracking option setting on. Multiple scans (more than twice) were performed and images with quality score greater than or equal to $7 / 10$ and without eye movement artifacts were preserved. The CC level is automatically defined as the distance between $10 \mu \mathrm{m}$ of the upper-bruch's membrane and $10 \mu \mathrm{m}$ of the lowerbruch's membrane. Manual adjustment was performed to ensure the scan was focused on the center of the macula and correct inaccurate automated segmentation. By referring to the reproducible automatic binarization methods reported previously $[8,11]$, we measured the percentage of black pixels representing absent or decreased flow signal within CC, known as FSV $(\%)[8,11]$, using image J software (ImageJ 1.52a, https://imagej.nih. gov/ij/ docs/) (Fig. 1).

All statistical tests were performed using SPSS version 23.0 (https:/www.ibm.com/a nalytics /spss-statisticssoftware). Categorized data are presented as frequency and percentage, and quantitative data are presented as mean \pm standard deviation. Shapiro-Wilk test was used to evaluate distribution. Student's t-test and one-way analysis of variance (ANOVA) were used for comparing variables between groups. Differences in proportions of gender were analyzed by $X^{2}$ [2] test. Pearson or Spearman method was used to analyze the correlation between FSV and age, and between FSV and
SFCT. A $P$-value $<0.05$ was considered statistically significant.

\section{Results}

A total of 28 affected eyes and 24 unaffected eyes from 26 CCSC patients, and 40 eyes of 20 age- and gendermatched healthy individuals were enrolled in this study. The baseline characteristics of the CCSC patients were as follows: $19(73.08 \%)$ males and 7 (26.92\%) females with an average age of $45.14 \pm 7.05$ years, and there were no statistically significant differences in age and gender between healthy individuals and CCSC patients ( $p=$ $0.084, p=0.899$, respectively). OCTA imaging with SRF, pigment epithelial detachment (PED), or RPE clumping was excluded from quantitative analysis of FSV (Fig. 2).

FSV Measurement was performed on both eyes of CCSC patients with complete resolution of SRF during follow-ups (Table 1). At 6-m follow-up, 2 affected eyes showed signs of reoccurred SRF and the patients complained of visual problems, while 7 affected eyes and 5 unaffected eyes were lost to follow-up. Significant improvement of BCVA was found at the last follow-up visit $(0.045 \pm 0.074$ LogMAR) compared to the initial $(0.235 \pm 0.137$ LogMAR) assessment $(p<0.001)$.

The FSV values of affected eyes were greater at $1-\mathrm{m}, 3-$ $\mathrm{m}$ and $6-\mathrm{m}$ follow-ups $(p<0.05$ for all), and the FSV values of unaffected eyes were significantly greater at baseline, $1-\mathrm{w}, 1-\mathrm{m}$ and $3-\mathrm{m}$ follow-ups ( $\mathrm{p}<0.05$ for all) than normal control eyes (Table 1). The FSV values in affected eyes were greater than those in unaffected eyes at $1-\mathrm{m}$ and 3-m post-treatment (Table 1). The Wilcoxon test results showed that the FSV value of affected eyes at 6-m followup was significantly greater than that at $1-\mathrm{m}$ follow-up $(p=0.036)$. The nonparametric Kruskal-Wallis test results showed that the FSV values of unaffected eyes showed no
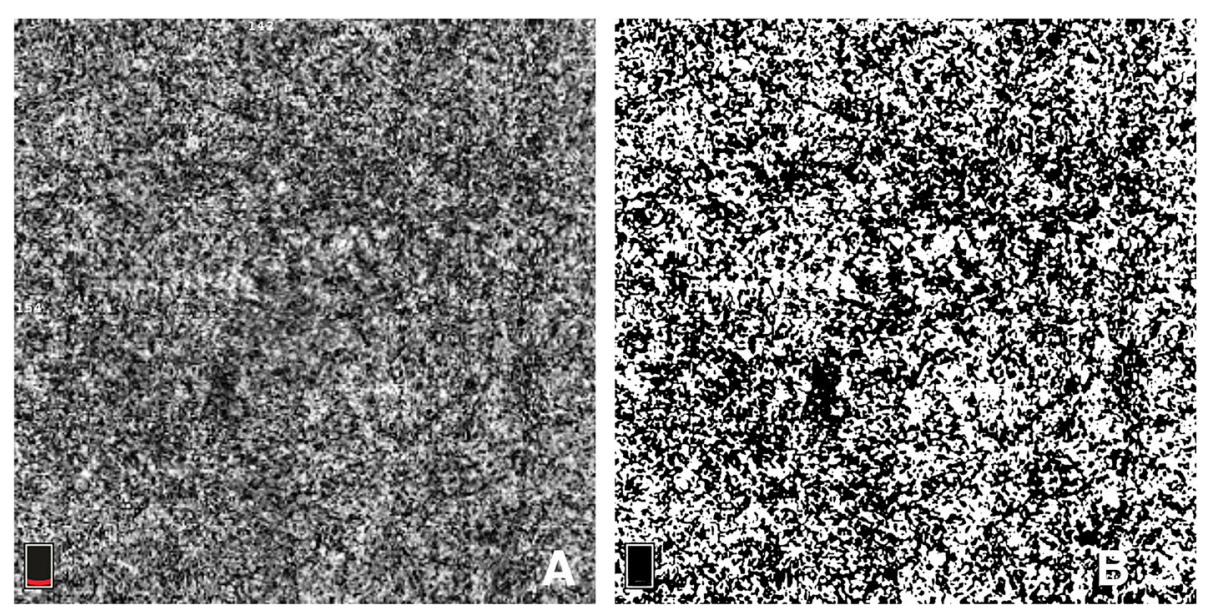

Fig. 1 The diagrammatic sketch of automatic quantitative binarization using ImageJ (b) of original image of en-face OCTA image of CC slab (a) 

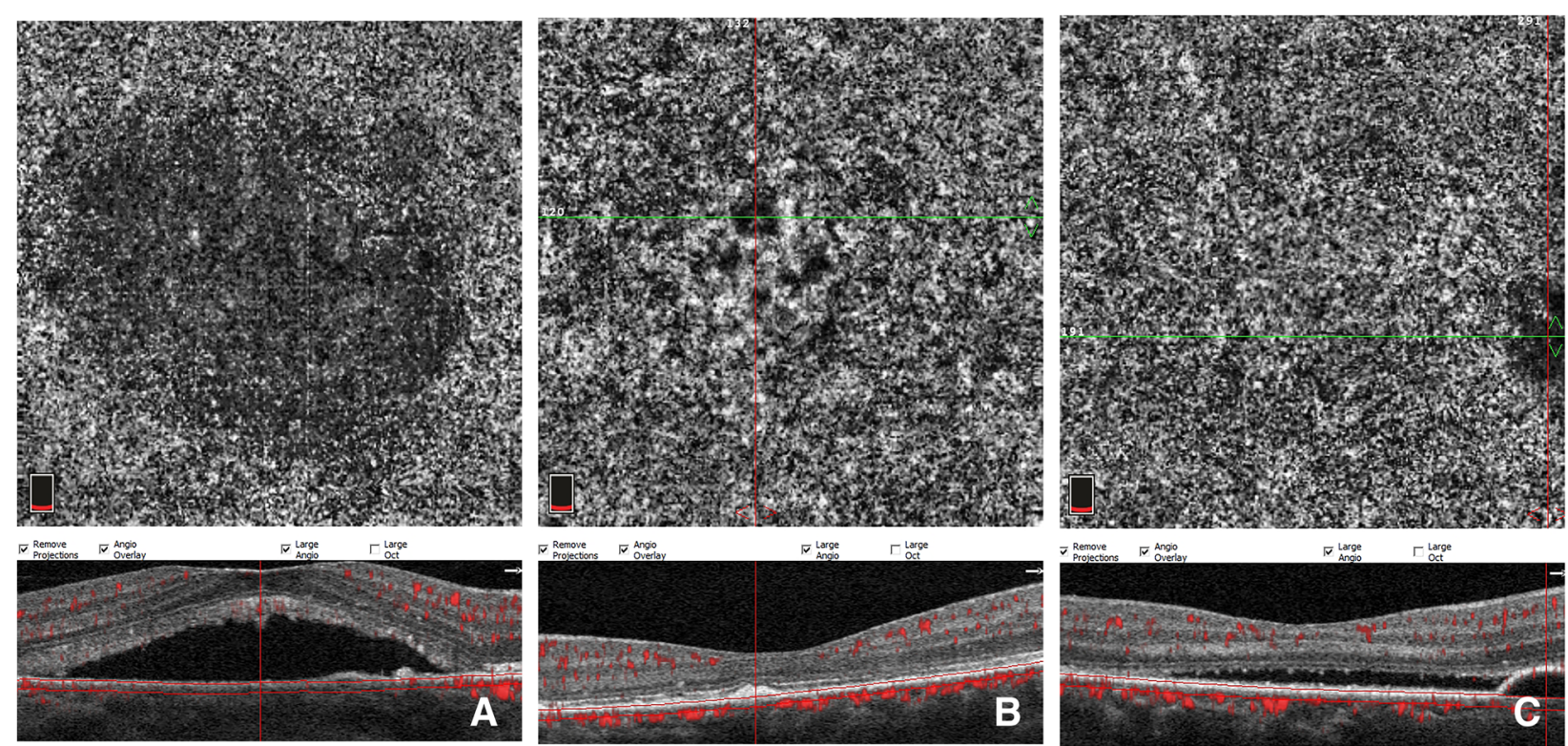

Fig. 2 En-face OCTA image of CC slab in affected eyes showing false positive FSV from subfoveal retinal detachment (a), RPE clumping (b) and PED (c) on the corresponding cross-sectional B-scan OCT.

significant differences between all subgroups of follow-ups $(0-\mathrm{m}, 1-\mathrm{w}, 1-\mathrm{m}, 3-\mathrm{m}$, and 6-m) $(p=0.674)$.

The SFCT values of both eyes of CCSC patients were greater than normal control eyes throughout the followup period in this cohort $(p<0.05$ for all), and significant difference was found between the affected and unaffected eyes of CCSC patients at baseline $(p=0.015)$ (Table 1). Nonparametric Kruskal-Wallis test results showed that the SFCT values of affected eyes in all subgroups $(0-\mathrm{m}, 1-\mathrm{w}, 1-\mathrm{m}, 3-\mathrm{m}$, and $6-\mathrm{m})$ were positive $(p=0.007)$, and the Post-hoc multiple comparisons within subgroups showed a significant difference in SFCT values between baseline and 3-m follow-up $(p=$
0.015). However, no statistically significant differences were observed in SFCT between all subgroups $(0-\mathrm{m}, 1-$ $\mathrm{w}, 1-\mathrm{m}, 3-\mathrm{m}$, and $6-\mathrm{m})$ of the unaffected eyes $(p=0.196)$ using nonparametric Kruskal-Wallis test.

Due to the inevitable shadowing artifacts of SRF, the FSV values at baseline and early stage after PDT were not quantified. Qualitative observation of CC patterns, as a supplement, showed that all the affected eyes demonstrated local or diffused flow signal attenuation, and dilation and tortuosity of $\mathrm{CC}$ at baseline. One week after PDT, three CC patterns of early response to PDT were identified by OCTA. Signs of recovery with increased blood flow signals and decreased dark areas were

Table 1 OCT and OCTA parameters of FSV and SFCT in patients with CCSC and healthy individuals

\begin{tabular}{|c|c|c|c|c|c|c|}
\hline \multirow[t]{2}{*}{ FSV } & \multirow{2}{*}{$\begin{array}{l}\text { normal subjects } \\
(\text { mean } \pm \text { SD) \% }\end{array}$} & \multicolumn{2}{|l|}{ CCSC eyes (mean \pm SD)\% } & \multicolumn{3}{|l|}{$p$-value } \\
\hline & & Affected eyes & Unaffected eyes & $\overline{P^{a}}$ & $P^{b}$ & $P^{c}$ \\
\hline $0 \mathrm{M}$ & $51.56 \pm 3.13 \%$ ( $n=40$ eyes $)$ & - & $53.63 \pm 3.09 \%$ ( $n=24$ eyes $)$ & - & 0.020 & - \\
\hline $1 \mathrm{M}$ & $51.56 \pm 3.13 \%$ & $55.98 \pm 3.82 \%(n=25$ eyes $)$ & $53.69 \pm 2.67 \%(n=24$ eyes $)$ & $<0.001$ & 0.010 & 0.005 \\
\hline $3 \mathrm{M}$ & $51.56 \pm 3.13 \%$ & $54.75 \pm 2.93 \%$ ( $n=27$ eyes $)$ & $53.41 \pm 2.38 \%$ ( $n=24$ eyes $)$ & $<0.001$ & 0.025 & 0.042 \\
\hline $6 \mathrm{M}$ & $51.56 \pm 3.13 \%$ & $53.56 \pm 3.28 \%$ ( $n=19$ eyes $)$ & $52.80 \pm 2.13 \%(n=19$ eyes $)$ & 0.035 & 0.098 & 0.758 \\
\hline \multirow[t]{2}{*}{ SFCT } & \multirow{2}{*}{$\begin{array}{l}\text { normal subjects } \\
(\text { mean } \pm \text { SD) } \mu \mathrm{m}\end{array}$} & \multicolumn{2}{|l|}{ CCSC eyes (mean \pm SD) $\mu \mathrm{m}$} & \multicolumn{3}{|l|}{$p$-value } \\
\hline & & Affected eyes & Unaffected eyes & $P^{a}$ & $P^{b}$ & $P^{c}$ \\
\hline OM & $267.73 \pm 99.18$ ( $n=40$ eyes $)$ & $447.29 \pm 82.28$ ( $n=28$ eyes $)$ & $397.33 \pm 70.57$ ( $n=24$ eyes $)$ & $<0.001$ & $<0.001$ & 0.004 \\
\hline $1 \mathrm{M}$ & $267.73 \pm 99.18$ & $387.50 \pm 93.77(n=28$ eyes $)$ & $370.62 \pm 74.63$ ( $n=24$ eyes $)$ & $<0.001$ & $<0.001$ & 0.484 \\
\hline $3 \mathrm{M}$ & $267.73 \pm 99.18$ & $375.30 \pm 95.45$ ( $n=28$ eyes $)$ & $356.46 \pm 73.06$ ( $n=24$ eyes $)$ & $<0.001$ & $<0.001$ & 0.407 \\
\hline $6 \mathrm{M}$ & $267.73 \pm 99.18$ & $375.76 \pm 104.15$ ( $n=21$ eyes) & $359.33 \pm 69.66$ ( $n=19$ eyes $)$ & $<0.001$ & $<0.001$ & 0.494 \\
\hline
\end{tabular}

CCSC chronic central serous chorioretinopathy, SFCT subretinal foveal choroidal thickness ( $\mu \mathrm{m})$; FSV (\%), proportion of total areas of flow signal voids; $\mathrm{P}^{\mathrm{a}}, p$-values for healthy subjects versus affected eyes using Mann-Whitney $\mathrm{U}$ test; $\mathrm{P}^{\mathrm{b}}, \mathrm{p}$-values for healthy eyes versus unaffected eyes using Mann-Whitney $\mathrm{U}$ test; $\mathrm{P}^{\mathrm{c}}, \mathrm{p}$ values for affected eyes versus unaffected eyes using Wilcoxon test 
observed in $21(75.0 \%)$ eyes (Fig. 3), while worsening local CC ischemia was observed in 6 (21.4\%) eyes (Fig. 5), and an extraordinarily dilated choriocapillary network or emerging dense network of neovascularization surrounded by foci of reduced flow signals within $\mathrm{CC}$ slab was observed in 1 (3.6\%) eye (Fig. 4).

Spearman correlation analysis showed that the mean value of FSV was positively correlated with age in the unaffected eyes of CCSC patients at onset $(r=0.469$, $p=0.021), 1-\mathrm{m}(\mathrm{r}=0.470, \mathrm{p}=0.021)$ and $3-\mathrm{m}$ followup $(\mathrm{r}=0.414, p=0.044)$ intervals and also in the eyes of healthy individuals $(\mathrm{r}=0.715, p<0.0001)$. However, no significant correlation was found between the values of FSV and SFCT.

\section{Discussion}

Using OCTA, quantitative analysis with automatic binarization method has been increasingly employed to analyze $\mathrm{CC}$ flow changes in some chorioretinal disorders, such as dry age-related macular degeneration and CSC, providing reproducible and objective data $[7,8$, 11]. Excluding the masking artifacts of SRF and RPE clumping, PED could help increase the reliability of the quantitative assessment in resolved CSC $[2,11]$. In previous studies, assessment of $\mathrm{CC}$ flow alterations in affected eyes showed a recovery after PDT $[7,13,14,16]$, which is consistent with our results of the decreased FSV at 6-m follow-up compared to that at 1-m followup. However, in this study, the FSA in affected eyes retained higher at 6-m follow-up compared with healthy controls, which indicated that $\mathrm{CC}$ attenuation might remain long after the treatment of half-dose PDT, contrary to previous OCTA studies which showed that CC flow returned to normal after half-dose PDT treatment [13, 14, 16]. A series of other follow-up investigations showed that reduced SFCT on EDI-OCT in affected eyes remained higher than healthy controls [1], and choroidal vascular hypermeability on ICGA persisted in some cases even after the SRF had been resolved completely $[17,18]$. The remaining choroidopathy observed after half-dose PDT could potentially be attributed to the primary pathogenesis of CSC and/or the therapeutic effects of PDT.

It has been postulated that CSC may be an ocular condition caused by a systemic disease that involves choroidal circulation [1], which could be potentially supported by previous results of some systemic risk factors [1] and abnormalities of CC and SFCT in selfresolved CSC eyes and unaffected fellow eyes [11, 1921]. Similarly, our study demonstrated that the FSV values of unaffected eyes of CCSC were higher than those in healthy individuals, and remained unchanged over time. As proposed by Nicolo, M [19],. these CC alterations in asymptomatic eyes might be in the early stage of the same condition of CSC $[2,11,19]$. Previous studies also detected punctated hyperfluorescent spots using ICGA in most contralateral eyes of CSC and PCV, which might be a subclinical manifestation of increased choroidal hypermeability and intrachoroidal hydrostatic pressure $[17,22]$. And these zones on OCTA with reduced CC flow have been found to be anatomically correlated with pathologically dilated Haller layer vessels on EDI-OCT. [2] In our study, the FSV values of affected eyes were significantly higher at $1-\mathrm{m}$ and $3-\mathrm{m}$ followups, but not at 6-m follow-up than unaffected eyes. We speculated that the abnormal vascular situation in the CC layer in affected eyes of CCSC might recover to a subclinical condition equivalent to that of the contralateral unaffected eyes, which needs to be validated by longitudinal investigations with larger sample size.

In order to evaluate the early $\mathrm{CC}$ alterations in response to half-dose PDT in CCSC, qualitative observation was performed and three CC patterns at one week after PDT were documented in the present study.

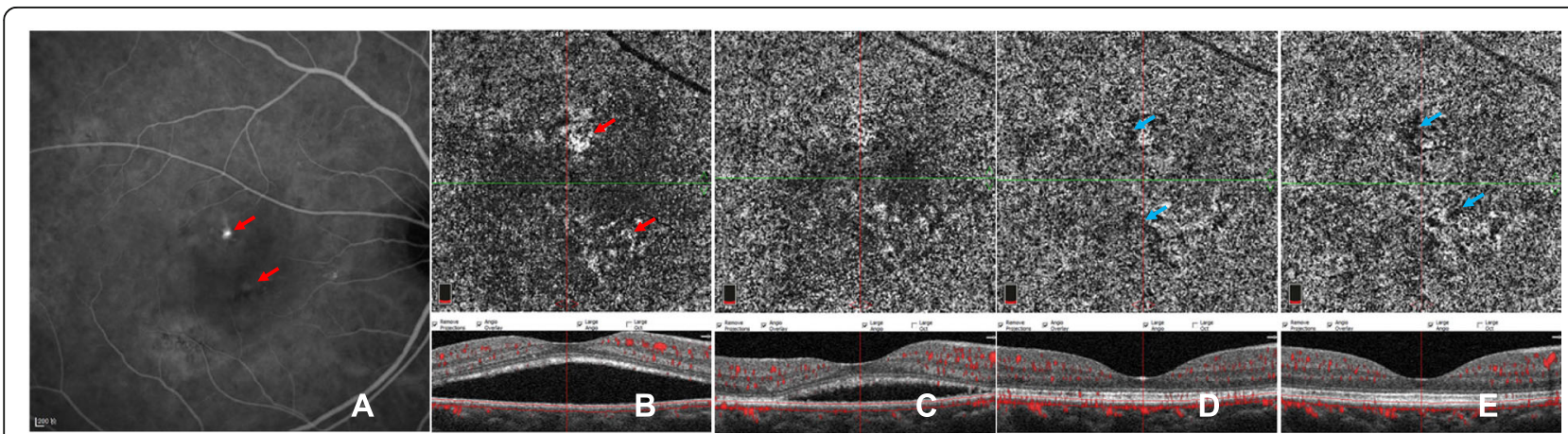

Fig. 3 Images of a case showing CC pattern of gradually increasing flow signals after PDT. ICGA (a) of baseline reveals partial choriocapillary hyperpermeability (red arrow) and focal hypo-fluorescent areas (blue arrow). En-face OCTA (b-e) of CC slab and the corresponding cross-sectional B-scan OCT (b-e) demonstrated the CC flow changes with time. Dilatation of CC (red arrow) accompanied by dark areas (blue arrow) can be seen at baseline (b). Recovery sign of increasing flow signals and decreasing dark areas was found at 1 week (c) after half-dose PDT and at the following 1-m (d), 3-m and 6-m (e) follow-ups, while foci of dark areas (blue arrows) remained 


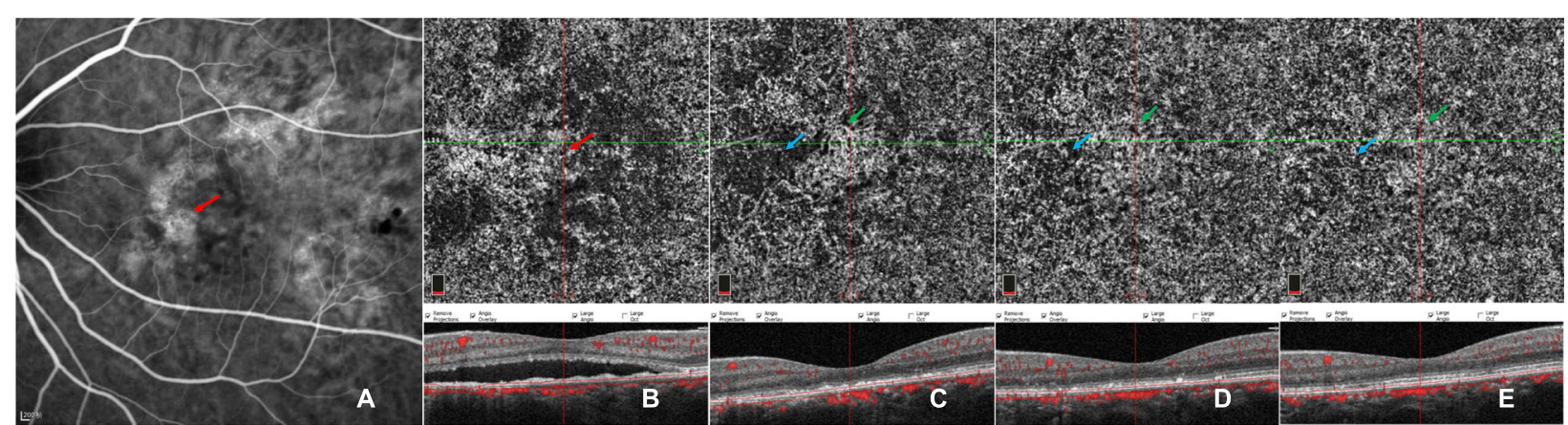

Fig. 4 Images of a case showing CC pattern of transient network of neovascularization. ICGA (a) of baseline revealed widespread lesions of choriocapillary hyperpermeability (red arrow) with hypo-fluorescent areas within them. En-face OCTA (b-e) of CC slab and the corresponding cross-sectional B-scan OCT (b-e) demonstrated the CC flow changes with time. Local dialed CC patterns in macular region surrounded by defused flow signal void were noticeable at baseline (b). An emerging network of neovascularization (green arrow) accompanying foci of reduced flow signals (blue arrow) was observed at 1 week after half-dose PDT (c), which gradually subsided (green arrows) during subsequent follow-ups of 1 month (d), 3 months and 6 months (e) while focally recovering with CC perfusion (blue arrow)

Among the affected eyes, $75.0 \%$ (21/28) showed recovering signs of increased flow signals and decreased dark areas (Fig. 3), while $21.4 \%$ (6/28) exhibited worse CC ischemia (Fig. 5), which we have previously documented as transient CC ischemia in another cohort [13]. Demircan et al. [16] showed that the transient CC ischemia may occur as early as three days after half-dose PDT. Furthermore, 3.6\% (1/28) of the affected eyes demonstrated transient appearance of exuberant neovascularization network within CC level (Fig. 4). Using OCTA, the direct action of PDT on the CC occlusion could be visualized in vivo during follow-up $[13,14,16]$. Posttreatment choroidal hypoperfusion was largely reported with evidence of hypoperfusion on traditional angiography [23] in the treatment of neovascular age-related macular degeneration with full PDT, which might be related to the preferential aggregation of verteporfin in the lesions [23].
The repair mechanisms of surviving endothelial cells and the recanalization processes of novel channels within previously occluded capillaries after PDT treatment remain unclear [1]. In addition to the possibility that CC flow recovers from the released pressure of decreased SFCT owing to the therapeutic effect of PDT $[2,11]$, it can also be speculated that the damaged choroidal endothelial cells and RPE cells in PDT-treated areas may contribute to the release of VEGF [24, 25], and an imbalanced stimulatory and inhibitory condition for neovascularization formation could be compromised by PDT-related hypoxia and ischemia $[25,26]$. However, the process of recanalization could, to some extent, contribute to the formation of CNV [26]. Particularly, two eyes with early CC ischemia, in this cohort, exhibited transient (Fig. 4) and persistent (Fig. 5) appearance of type I CNV, consisting with the morphologic characteristics of neovascularization networks within CC level in previous OCTA-related studies [20, 27, 28]. However, it

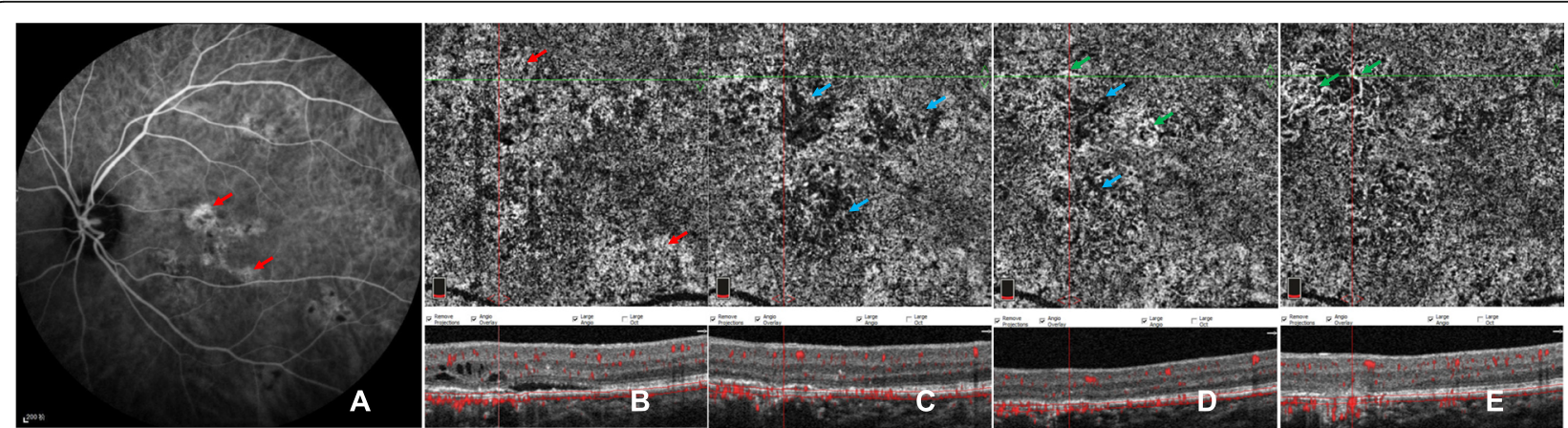

Fig. 5 Images of a case showing CC pattern of worse CC ischemia followed by persistent type I CNV. ICGA (a) of baseline revealed multifocal choriocapillary hyperpermeability (red arrows). En-face OCTA (b-e) of CC slab and the corresponding cross-sectional B-scan OCT (b-e) demonstrated the CC flow changes with time. Defused dilatation of CC (red arrow) (b) can be detected at baseline, with punctate dark areas within the lesions. Local worse CC ischemia (blue arrows) was found at 1 week after half-dose PDT (c), combined with dynamic changes of neovascularization of sprouts (d) at 1-m follow-up, and grew with loose network of CNV (green arrows) during follow-ups of 3 months and 6 months (e) while focally recovering with CC perfusion (blue arrow) 
is controversial that these suspected secondary $\mathrm{CNV}$ within $\mathrm{CC}$ level may contribute to the $\mathrm{CC}$ atrophy and the anterior displacement of medium-sized choroidal vessels with segmentation artifacts that masquerade as CNV [24]. Moreover, type I CNV has been well documented as the most common subtype of secondary $\mathrm{CNV}$ in the natural course of CSC [1, 6, 29], which should also be taken into account while studying PDT-related CNV [30]. What makes it more controversial is that most of the previous studies of CSC related $\mathrm{CNV}$ were based on patients with heterogeneous treatment histories of PDT or laser photocoagulation [20, 28-30]. Longitudinal OCTA observation would thus help to comparatively follow these lesions to better understand how they behave.

There are some limitations in our study including its retrospective nature, the relatively small number of subjects. Considering the shortage of quantitative method of binarization that limited our ability to assess FSV in affected eyes at the time point of pre-PDT and at the early stage of post-PDT owing to the shadowing artifacts of unresolved subretinal fluid [7], we only compared the FSV values between the time points of $1 \mathrm{~m}$ and $6 \mathrm{~m}$ after PDT using Wilcoxon test. We ought to record the change terms of exact number of lines or letters in BCVA in this study, which may be more clinically relevant. Our results may underestimate the occurrence of these transient alterations of ischemia and neovascularization occurred within two intervals or outside the imaging area of OCTA. We believe that the actual value of FSV may be higher since patients with SRF were excluded in quantitative assessment, and the choriocapillaris in these patients was certainly abnormal. Although the FSV value was positively correlated with age in healthy controls in consistence with aging physiological changes of $\mathrm{CC}[8,11]$, the dynamic nature of $\mathrm{CC}$ flow changes with time should also be taken into consideration in analysis. In addition, the correlation between SFCT and FSV has not been established in this cohort, nor in other reports [31]. It might be due to the relatively small sample size, or that SFCT generally assesses choroidal thickness and both pathologically dilated vessels and increased stromal contribute to the increase of SFCT. Future studies should further evaluate the correlation of FSV with other more specific indicators such as choroidal vascularity index (CVI) [3].

\section{Conclusions}

In summary, we observed that abnormal CC flow attenuation remained in clinically resolved eyes of CCSC patients treated with half-dose PDT. It is noteworthy that half-dose PDT, as a safe therapeutic method for CCSC, could potentially worsen choroidal ischemia, which is a pathophysiologic cause of neovascularization progressions in some cases, while OCTA is helpful in identifying these $\mathrm{CC}$ changes and following these lesions over time to better understand how they progress.

\section{Abbreviations}

OCTA: Optical coherence tomography angiography; CC: Choriocapillaris; CCSC: Chronic central serous chorioretinopathy; SRF: Subretinal fluid; RPE: Retinal pigment epithelial; PED: Pigment epithelial detachment; PDT: Photodynamic therapy; FSV: Flow signal voids; EDI-OCT: Enhanced depth imaging optical coherence tomography; SFCT: Subfoveal choroidal thickness; ICGA: Indocyanine green angiography; CNV: Choroidal neovascularization

\section{Acknowledgements}

We would like to thank professor TH, CZC and HMZ for their support and help on our work.

\section{Authors' contributions}

$J \mathrm{~J}$ and LL contributed to patient care. JJL, CZC, LL, ZHZY, LH, and HMZ contributed to literature search, data collection, data analysis, and data interpretation. JJL and CZC drafted and revised the manuscript. All authors have read and approved the final manuscript.

\section{Funding}

Not applicable.

\section{Availability of data and materials}

The datasets used and/or analyzed during the current study are available from the corresponding author on reasonable request.

\section{Ethics approval and consent to participate}

This retrospective study was approved by the Renmin Hospital of Wuhan University and was conducted in compliance with the Declaration of Helsinki. All participants provided their written informed consent.

\section{Consent for publication}

Not applicable.

\section{Competing interests}

Not applicable.

Received: 20 November 2019 Accepted: 30 September 2020

Published online: 07 October 2020

\section{References}

1. Daruich A, Matet A, Dirani A, et al. Central serous chorioretinopathy: recent findings and new physiopathology hypothesis. Prog Retin Eye Res. 2015;48: $82-118$

2. Gal-Or O, Dansingani KK, Sebrow D, Dolz-Marco R, Freund KB. Inner Choroidal Flow Signal Attenuation in Pachychoroid Disease: optical coherence tomography angiography. Retina. 2018;38(10):1984-92.

3. Wei X, Sonoda S, Mishra C, et al. Comparison of Choroidal vascularity markers on optical coherence tomography using two-image Binarization techniques. Invest Ophth Vis Sci. 2018;59(3):1206-11..

4. Lai FH, Ng DS, Bakthavatsalam M, et al. A multicenter study on the longterm outcomes of half-dose photodynamic therapy in chronic central serous Chorioretinopathy. Am J Ophthalmol. 2016;170:91-9.

5. Schmidt-Erfurth $U$, Laqua H, Schlotzer-Schrehard U, Viestenz A, Naumann GO. Histopathological changes following photodynamic therapy in human eyes. Arch Ophthalmol. 2002;120(6):835-44.

6. Borrelli E, Sarraf D, Freund KB, Sadda SR. OCT angiography and evaluation of the choroid and choroidal vascular disorders. Prog Retin Eye Res. 2018;67: 30-55.

7. Lauermann JL, Eter N, Alten F. Optical coherence tomography angiography offers new insights into Choriocapillaris perfusion. Ophthalmologica. 2018; 239(2-3):74-84

8. Spaide RF. Choriocapillaris flow features follow a power law distribution: implications for characterization and mechanisms of disease progression. Am J Ophthalmol. 2016;170:58-67.

9. Teussink MM, Breukink MB, Grinsven MJ, Hoyng CB, Klevering BJ. OCT angiography compared to fluorescein and indocyanine green ngiography in chronic central serous chorioretinopathy. Invest Ophthalmol Vis Sci. 2015; 56(9):5229-37 
10. Quaranta-El Maftouhi M, El Maftouhi A, Eandi CM. Chronic central serous chorioretinopathy imaged by optical coherence tomographic angiography. Am J Ophthalmol. 2015;160(3):581-7 e581.

11. Rochepeau C, Kodjikian L, Garcia MA, et al. Optical coherence tomography angiography quantitative assessment of Choriocapillaris blood flow in central serous Chorioretinopathy. Am J Ophthalmol. 2018; 194:26-34.

12. Ogawa Y, Maruko I, Koizumi H, lida T. Quantification of Choroidal Vasculature by High-Quality Structure En Face Swept-Source Optical Coherence Tomography Images in Eyes with Central Serous Chorioretinopathy. Retina. 2018;40(3):529-36.

13. Xu Y, Su Y, Li L, Qi H, Zheng H, Chen C. Effect of photodynamic therapy on optical coherence tomography angiography in eyes with chronic central serous Chorioretinopathy. Ophthalmologica. 2017;237(3):167-72.

14. Nassisi M, Lavia C, Alovisi C, Musso L, Eandi CM. Short-Term Choriocapillaris Changes in Patients with Central Serous Chorioretinopathy after Half-Dose Photodynamic Therapy. Int J Mol Sci. 2017;18(11):2468. https://doi.org/10. 3390/ijms18112468.

15. Kraus MF, Liu JJ, Schottenhamml J, et al. Quantitative 3D-OCT motion correction with tilt and illumination correction, robust similarity measure and regularization. Biomed Opt Express. 2014;5(8):2591-613.

16. Demircan A, Yesilkaya C, Alkin Z. Early choriocapillaris changes after halffluence photodynamic therapy in chronic central serous chorioretinopathy evaluated by optical coherence tomography angiography: preliminary results. Photodiagn Photodyn Ther. 2018;21:375-8.

17. Tsujikawa A, Ojima Y, Yamashiro K, et al. Punctate hyperfluorescent spots associated with central serous chorioretinopathy as seen on indocyanine green angiography. Retina. 2010;30(5):801-9.

18. lida T, Kishi S, Hagimura N, Shimizu K. Persistent and bilateral choroidal vascular abnormalities in central serous chorioretinopathy. Retina. 1999; 19(6):508-12.

19. Nicolo M, Rosa R, Musetti D, Musolino M, Saccheggiani M, Traverso CE. Choroidal vascular flow area in central serous Chorioretinopathy using swept-source optical coherence tomography angiography. Invest Ophth Vis Sci. 2017;58(4):2002-10.

20. Mandadi SKR, Singh SR, Sahoo NK, et al. Optical coherence tomography angiography findings in fellow eyes of choroidal neovascularisation associated with central serous chorioretinopathy. Br J Ophthalmol. 2019. https://doi.org/10.1136/bjophthalmol-2018-313576..

21. Kang NH, Kim YT. Change in subfoveal choroidal thickness in central serous chorioretinopathy following spontaneous resolution and low-fluence photodynamic therapy. Eye (Lond). 2013;27(3):387-91.

22. Park S, Kim B, Park K, Woo S. Punctate hyperfluorescence spot as a common choroidopathy of central serous chorioretinopathy and polypoidal choroidal vasculopathy. Am J Ophthalmol. 2014;158(6):1155-63.

23. Schmidt-Erfurth U, Kiss C, Sacu S. The role of choroidal hypoperfusion associated with photodynamic therapy in neovascular age-related macular degeneration and the consequences for combination strategies. Prog Retin Eye Res. 2009;28(2):145-54.

24. Chhablani J, Pichi F, Silva R, et al. Antiangiogenics in Choroidal neovascularization associated with laser in central serous Chorioretinopathy. Retina. 2016;36(5):901-8.

25. Schmidt-Erfurth U, Schlotzer-Schrehard U, Cursiefen C, Michels S, Beckendorf A, Naumann GO. Influence of photodynamic therapy on expression of vascular endothelial growth factor (VEGF), VEGF receptor 3, and pigment epithelium-derived factor. Invest Ophthalmol Vis Sci. 2003;44(10):4473-80.

26. Schlotzer-Schrehardt U, Viestenz A, Naumann GO, Laqua H, Michels S, Schmidt-Erfurth U. Dose-related structural effects of photodynamic therapy on choroidal and retinal structures of human eyes. Graefes Arch Clin Exp Ophthalmol. 2002;240(9):748-57.

27. Moussa M, Leila M, Khalid H, Lolah M. Detection of Silent Type I Choroidal Neovascular Membrane in Chronic Central Serous Chorioretinopathy Using En Face Swept-Source Optical Coherence Tomography Angiography. J Ophthalmol. 2017;2017:6913980. https://doi.org/10.1155/2017/6913980.

28. Spaide RF. Optical coherence tomography angiography signs of vascular Abnormalization with Antiangiogenic therapy for Choroidal neovascularization. Am J Ophthalmol. 2015;160(1):6-16.

29. Shiragami C, Takasago Y, Osaka $R$, et al. Clinical features of central serous Chorioretinopathy with type 1 Choroidal neovascularization. Am J Ophthalmol. 2018;193:80-6.
30. Wu JS, Chen SN. Optical coherence tomography angiography for diagnosis of Choroidal neovascularization in chronic central serous Chorioretinopathy after photodynamic therapy. Sci Rep. 2019;9(1):9040.

31. Yun C, Huh J, Ahn SM, et al. Choriocapillaris flow features and choroidal vasculature in the fellow eyes of patients with acute central serous chorioretinopathy. Graefes Arch Clin Exp Ophthalmol. 2019;257(1):57-70.

\section{Publisher's Note}

Springer Nature remains neutral with regard to jurisdictional claims in published maps and institutional affiliations.
Ready to submit your research? Choose BMC and benefit from:

- fast, convenient online submission

- thorough peer review by experienced researchers in your field

- rapid publication on acceptance

- support for research data, including large and complex data types

- gold Open Access which fosters wider collaboration and increased citations

- maximum visibility for your research: over $100 \mathrm{M}$ website views per year

At $\mathrm{BMC}$, research is always in progress.

Learn more biomedcentral.com/submissions 